\title{
Collarette Region Recognition based on Wavelets and Direct Linear Discriminant Analysis
}

\author{
Akanksha Joshi \\ Center for Development of \\ Advanced Computing \\ Mumbai, India
}

\author{
Abhishek Gangwar \\ Center for Development of \\ Advanced Computing \\ Mumbai, India
}

\author{
Zia Saquib \\ Center for Development of \\ Advanced Computing \\ Mumbai, India
}

\begin{abstract}
Iris recognition is seen as a highly reliable biometric technology. The performance of iris recognition is severely impacted when encountering poor quality images. The selection of the features subset and the classification is an important issue for iris biometrics. Here, we explored the contribution of collarette region in identifying a person. We applied five level haar wavelet decomposition for collarette region feature extraction and used the second level approximation coefficients combined with fifth level vertical coefficients for better accuracy. Then, we applied Direct Linear Discriminant Analysis (DLDA) to produce discriminative low-dimensional feature vectors. The approach is evaluated on CASIA Iris Interval database and we achieved $98.96 \%$ accuracy using the collarette region which is a significant improvement in the performance of recognition.
\end{abstract}

\section{General Terms}

Pattern Recognition, Security, Biometric person recognition, Iris recognition, Biometric Identification.

\section{Keywords}

Collarette Feature Extraction, Wavelet Transform.

\section{INTRODUCTION}

With increase in terrorism and illegal acts, there is a growing demand for more secure and reliable identification in our society that can replace the traditional means of identification. Biometric technologies, based on recognition of humans based on behavioral or physiological characteristics, promises to be an effective solution. Biometric recognition can be described as automated methods to accurately recognize individuals based on distinguishing physiological and/or behavioral traits. It is a subset of the broader field of the science of human identification. Biometrics offers the means to identify individuals without requiring that they carry ID cards and badges or memorize passwords. Examples of biometric technologies include fingerprint recognition, face recognition, iris recognition and many others. The parts of body and behavior have been used for years as a means of person recognition and authentication. For example, fingerprint has been used for a long time in security and access applications.

Different biometric technologies work optimally based on a variety of factors. In order to make a decision of which biometric technologies satisfy the application requirements, a variety of factors need to be assessed. Factors for consideration typically include accuracy of a specific technology under different environment conditions, user acceptance, and the costs of implementation and operation.

In comparison to other biometric features such as face, fingerprint, retina, and hand geometry, iris is seen as a highly reliable biometric technology because of its stability, and high degree of variation between individuals.

The iris is seen as a highly reliable and accurate biometric technology because each human being is characterized by unique irises that remain relatively stable over the life period. Iris is present in the form of ring around pupil of a human eye in all the human beings. Its complex pattern contains many distinctive features such as arching ligaments, crypts, radial furrows, pigment frill, papillary area, ciliary area, rings, corona, freckles and zigzag collarette [1][2] which gives a unique set of feature for each human being, even irises of identical twins are different. The iris is more easily imaged than retina; it is extremely difficult to surgically tamper iris texture information and it is possible to detect artificial irises.

Surface of the iris is composed of two regions, the central pupillary zone and the outer ciliary zone. The collarette is the border between these two regions (Figure 1). The collarette region is less sensitive to the pupil dilation and usually unaffected by the eyelashes and eyelids [15].

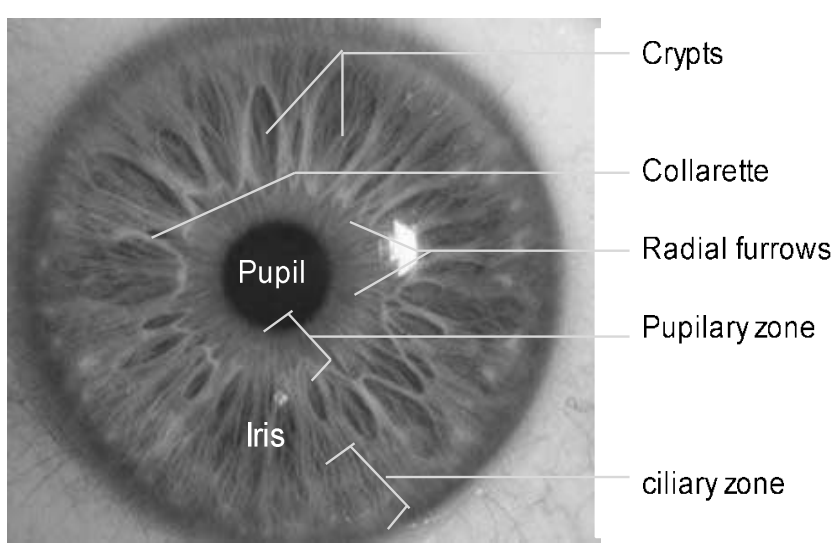

Figure 1. Pupillary zone, collarette and ciliary area

In this paper, we propose a new feature extraction technique using wavelets [11] combined with DLDA [7] to extract discriminative low-dimensional feature vectors from collarette region. For wavelet feature extraction, we tried combinations of different coefficients from level one to level five and selected only those which provided the most accurate results. We evaluated our approach on images from CASIA database [12]. The images are captured in near infrared illumination. First we applied wavelets on the iris region and achieved an accuracy of $93.26 \%$, whereas we achieved accuracy of $98.96 \%$ when we extracted collarette features using wavelet combined with DLDA.

The remainder of this paper is as follows. Section 2 provides a brief overview of iris recognition, then edge detection, Hough 
transform, Wavelet Transform, and DLDA. Section 3 explains our proposed approach. Section 4 gives experimental evaluation $\&$ results and section 5 provides conclusion of our work.

\section{IRIS RECOGNITION}

Iris recognition starts with capturing the eye and localizing the iris region. Classical iris recognition systems, e.g., Daugman's and Wildes', need the users to adjust their eye positions in order to capture their irises [3]. Furthermore, existing systems require users to be close to the capturing apparatus [4]. A common observation about eye images is that the iris region is brighter than the pupil and darker than the sclera. As a result, almost all approaches to iris localization are based on the intensity gradient or edge information. These methods depend heavily on the strong intensity contrast between the pupil and iris and between the iris and sclera. Wildes [13] proposed iris segmentation by edge detection by canny filter followed by circular Hough transform. It is based on the assumption that pupillary and limbic boundaries are circular and eyelids are parabolic in shape. Since variations in the eye, like optical size of the iris, position of pupil in the iris, and the iris orientation changes from person to person, it is required to normalize the iris image, so that the representation is common to all, with similar dimensions. Normalization process involves unwrapping the iris and converting it into its polar equivalent. It is done using Daugman's Rubber sheet model [2].

The iris features of a human eye may be extracted using wavelet transform. We can use wavelets for multiresolution decomposition analysis for iris of a human eye. Wavelets are a powerful tool and have been applied earlier for image compression and texture classification. The wavelet analysis can be done by successive low pass and high pass filtering of an image. Once features are generated, comparison of the bit patterns is done to check if the two images belong to the same person. Euclidean Distance is used for this comparison

Here is the brief overview of the techniques used in our approach.

\subsection{Canny Edge Detector}

Detection of iris edges includes inner (with pupil) and outer (with sclera) edges which are located by finding the edge image using the Canny edge detector [6].

The Canny detector creates binary edge map correspondent to the identified edges in the grayscale image. It starts with finding a gradient map for each image pixel. Then nonmaximum suppression is applied in following manner. For a pixel imgrad(x,y), in the gradient image, and given the orientation theta $(\mathrm{x}, \mathrm{y})$, the edge intersects two of its 8 connected neighbors. The point at $(\mathrm{x}, \mathrm{y})$ is a maximum if its value is not smaller than the values at the two intersection points. Further, the hysterisis thresholding process uses two predefined values to classify some pixels as edge or non-edge. In next step, edges are recursively extended to those pixels that are neighbors of other edges and with gradient amplitude higher than a lower threshold. Now Hough transform [5] is applied to detect circles in the edge image.

\subsection{Hough Transform}

For every edge pixel, the points on the circles surrounding it at different radii are taken, and their weights are increased if they are edge points too, and these weights are added to the accumulator array. Thus, after all radii and edge pixels have been searched, the maximum from the accumulator array is used to find the center of the circle and its radius. The Hough transform is performed for the iris outer boundary using the whole image, and then performed for the pupil only, instead of the whole eye, because the pupil is always inside the iris.

$\mathrm{H}\left(\mathrm{x}_{\mathrm{c}}, \mathrm{y}_{\mathrm{c}}, \mathrm{r}\right)=\sum_{j=1}^{n} \mathrm{~h}\left(\mathrm{x}_{\mathrm{j}}, \mathrm{y}_{\mathrm{j}}, \mathrm{x}_{\mathrm{c}}, \mathrm{y}_{\mathrm{c}}, \mathrm{r}\right)$,

Where

$h\left(x_{j}, y_{j}, x_{c}, y_{c}, r\right)= \begin{cases}1 & \text { if } g\left(x_{j}, y_{j}, x_{c}, y_{c}, r\right)=0 ; \\ 0 & \text { otherwise. }\end{cases}$

With

$$
g\left(x_{j}, y_{j}, x_{c}, y_{c}, r\right)=\left(x_{j}-x_{c}\right)^{2}+\left(y_{j}-y_{c}\right)^{2}-r^{2} .
$$

For each edge point (xi, yi), this function returns zero for every parameter triple $(\mathrm{xc}, \mathrm{yc}, \mathrm{r})$ that represents a circle through that point. Parameter triple (xc, yc, r) represents the contour of interest for which $\mathrm{H}$ is maximum.

\subsection{Rubber sheet model by Daugman}

The rubber sheet model [2] assigns to each point on the iris, regardless of its size and pupillary dilation, a pair of real coordinates $(r, \theta)$, where $\mathrm{r}$ is on the unit interval $[0,1]$ and

$\theta$ is an angle in $\left[0,360^{\circ}\right]$. The remapping of the iris image $\mathrm{I}(\mathrm{x}, \mathrm{y})$ from raw cartesian coordinates $(\mathrm{x}, \mathrm{y})$ to the dimensionless non concentric polar coordinate system $(r, \theta)$ can be represented as:

$$
I(x(r, \theta), y(r, \theta)) \rightarrow I(r, \theta)
$$

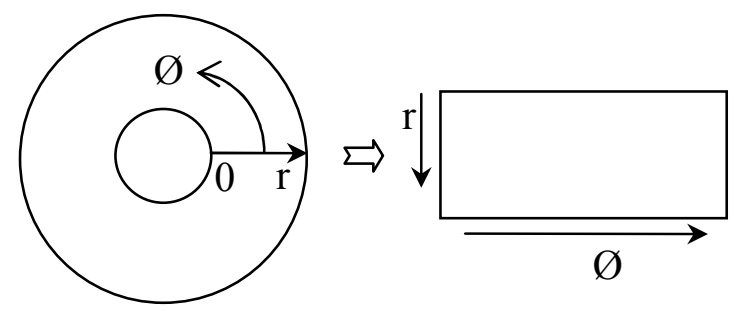

Figure 2. Normalization process

Where $x(r, \theta)$ and $y(r, \theta)$ are defined as linear combinations of both the set of papillary boundary points $\left.\left(x_{p}(\theta)\right), y_{p}(\theta)\right)$ and the set of collarette boundary points $\left.\left(x_{c}(\theta)\right), y_{c}(\theta)\right)$ as:

$\left\{\begin{array}{l}x(r, \theta)=(1-r) * x_{p}(\theta)+r * x_{c}(\theta) \\ y(r, \theta)=(1-r) * y_{p}(\theta)+r * y_{c}(\theta)\end{array}\right.$ 


\subsection{Wavelet}

Wavelets [11] are used for multiresolution analysis of signals and have been a useful tool for analyzing patterns in images. Wavelets can be applied at different scales or levels. Level one wavelet decomposition will yield four sub-images or bands; approximate, vertical, horizontal and detail. The approximate sub-band extracts the low level details from the image (LL), whereas vertical sub-band extracts the high frequencies in vertical direction and low frequencies in the horizontal direction which is basically the HL component of the sub image. The horizontal component (LH) extracts the high frequencies in horizontal direction and low frequencies in vertical direction and the diagonal part contains the high frequencies in horizontal as well as vertical direction. The second level wavelet decomposition is obtained by applying the wavelet decomposition to the approximate sub-image obtained from level one decomposition. Thus wavelet decomposition can be obtained at various levels by recursively applying the decomposition on the approximate part of the image obtained in the previous level. Two level wavelet decomposition is shown in Fig. 3

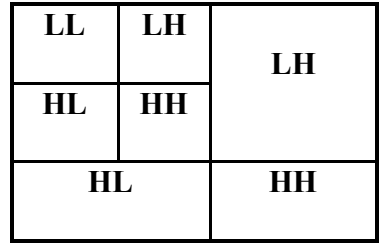

Figure 3. Two level wavelet decomposition.

\subsection{Direct Linear Discriminant Analysis (DLDA)}

Direct Linear Discriminant Analysis [7] is a well known classification technique and has been applied over face to reduce the feature dimensions. It basically transforms the data to lower dimensions, without losing the discrimination information. Previously, people applied PCA + LDA approach [8] but applying PCA tend to lose the discriminatory information among classes and thus yielding low accuracy. LDA aims at maximizing the ratio of between class scatter $S_{b}$ and within class scatter $S_{w}$. It discards the null space of $S_{w}$, which contains the most discriminatory information according to Chen et al. [9]. The key idea of DLDA algorithm is to discard the null space of $\mathrm{S}_{b}$, which contains no useful information rather than discarding the null space of $S_{w}$, which contains the most discriminative information. This can be done by first diagonalizing $\mathrm{S}_{b}$ and then diagonalizing $S_{w}$. The whole DLDA algorithm is outlined below.

1. First diagonalize the $\mathrm{S}_{b}$ matrix, such that

$$
V^{T} S_{b} V=D
$$

It involves finding the eigenvectors of matrix $\mathrm{S}_{b}$ and matrix D contains the corresponding eigen values. We discard those values from $\mathrm{V}$ which contains eigen values corresponding to 0 such that

$$
Y^{T} S_{b} Y=D_{b}>0
$$

Where $\mathrm{Y}$ is $\mathrm{n} * \mathrm{~m}$ matrix ( $\mathrm{n}$ is the feature dimension) and contains first $\mathrm{m}$ columns from $\mathrm{V}$ and $D_{b}$ is $\mathrm{m}^{*} \mathrm{~m}$ matrix corresponding to non-zero eigen values.

$$
\begin{aligned}
& \text { 2. Let } Z=Y D_{b}^{-1 / 2} \text {, where } \\
& \qquad \mathrm{Z}^{T} \mathrm{~S}_{b} \mathrm{Z}=\mathrm{I}
\end{aligned}
$$

Where, $Z$ unitizes $\mathrm{S}_{b}$ and reduces dimensionality from $\mathrm{n}$ to $\mathrm{m}$.

3. Now we need to diagonalize $\mathrm{Z}^{T} \mathrm{~S}_{w} \mathrm{Z}$ as,

$$
U^{T}\left(Z^{T} S_{w} Z\right) U=D_{w}
$$

4. Let $A=U^{T} Z^{T}$ diagonalizes both numerator and denominator in Fisher's criteria as,

$$
{ }^{T} S_{w^{A}}=D_{w}, A^{T} S_{b} A=I
$$

5. Thus, we get the final transformations as

$$
T=D_{b}^{-1 / 2} A X
$$

Where $\mathrm{X}$ is the feature vector extracted from the image and $\mathrm{T}$ is the reduced feature vector.

\subsection{Euclidean Distance}

The Euclidean Distance is a method to find similarity between two feature vectors. The Euclidean Distance is calculated by measuring the norm between two vectors as,

$$
D=\sqrt{(X 2-X 1)^{2}+(Y 2-Y 1)^{2}}
$$

\section{PROPOSED APPROACH}

In our approach, we segmented the pupil and iris region using the canny edge detector and Hough transform. The collarette region is concentric with the pupil and is restricted to a certain range within the iris [10]. Thus, we segmented the collarette part by choosing appropriate radius from the center of pupil towards the iris region. The collarette segmentation is shown in Fig.4. The extracted collarette region is normalized using rubber sheet model and wavelet decomposition is applied to extract features from the collarette region. We applied five level wavelet decomposition and utilized the 2nd (second) level approximate and 5th (fifth) level vertical coefficients only from the image. We have chosen the 2nd level approximate coefficients because they contain most of the information from the image. Fifth level coefficients provides the discriminatory information therefore, we also selected 5 th level vertical coefficients from the image. A five level wavelet decomposition of the collarette region is shown in Fig. 5. After extracting features using wavelets, we applied DLDA to get better discrimination among extracted wavelet features. DLDA reduces the dimensionality of the feature vector and also provide better discrimination among feature vectors. 


\section{EXPERIMENTAL RESULTS}

We evaluated our approach on CASIA Iris Interval database. It contains left and right eye iris images from 249 users. We tried different combinations of wavelet (Haar and Db4) coefficients from each level and selected the second level approximate and fifth level vertical coefficients.

\subsection{Experiment 1}

In first experiment, we applied wavelets on the iris region. We used euclidean distance for matching wavelet features of two images. We achieved an accuracy of $93.26 \%$ when we used $\mathrm{db} 4$ wavelet decomposition on iris region and $89.63 \%$ using haar wavelet, whereas we got an accuracy of $94.81 \%$ when using db4 wavelet and $89.56 \%$ with haar wavelet on collarette region.

\subsection{Experiment 2}

In second experiment, first we extracted iris features using wavelets (haar and db4) and then applied DLDA. We used five images per person for training and used Euclidean distance for matching images. We got an accuracy of $97.44 \%$ for iris region with $\mathrm{db} 4$ wavelet and $97.40 \%$ with haar wavelet. We achieved $98.96 \%$ accuracy when we used db4 wavelet and DLDA and an accuracy of $98.44 \%$ with haar wavelet and DLDA over the collarette region

We can observe from the results (given in Table I) that an approach combining wavelet and DLDA gives much better accuracy than wavelets alone. Moreover, db4 wavelet performs much better as compared to haar wavelet decomposition over iris region. It is also visible from Table I that we can utilize the collarette region instead of whole iris for identification because collarette region alone can give the comparable accuracy as given by iris. We observed almost the same or a bit higher accuracy from collarette region than whole iris region.

\section{CONCLUSION}

The performance of iris recognition is severely impacted when encountering poor quality image like occlusion by eyelashes and eyelids, therefore, in this paper we have used collarette region for person identification. We evaluated wavelets (haar and db4) combined with DLDA on collarette region. For wavelet feature extraction, we used the second level approximate and fifth level vertical coefficients as they provided the most relevant information required for identification. Experimentally we have shown that feature extraction based on wavelets combined with DLDA give better accuracy compared to wavelets alone. Also, it is visible from experiments that collarette region contains enough information needed for person identification.

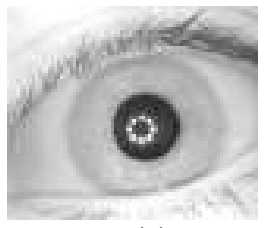

(a)

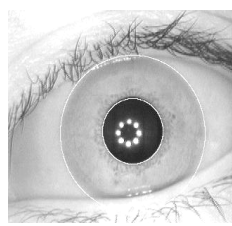

(b)

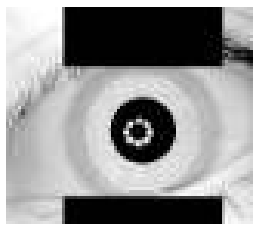

(c)
Figure 4. (a) Image from CASIA database (b) Iris segmented image (c) Collarette segmented image

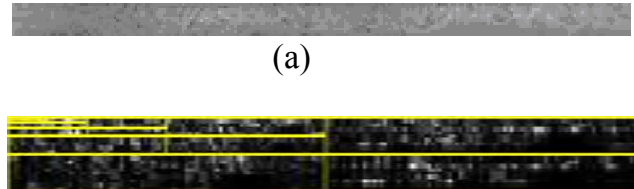

(b)

Figure 5. (a) Normalized collarette image (b) Five level wavelet decomposition for collarette portion of image

Table 1. Identification Accuracies

\begin{tabular}{|c|c|c|c|c|}
\hline \multirow{2}{*}{$\begin{array}{l}\text { Image } \\
\text { region } \\
\text { selected }\end{array}$} & \multicolumn{3}{|l|}{$\begin{array}{l}\text { Identification } \\
\text { accuracy (\%) } \\
\text { using wavelet }\end{array}$} & \multicolumn{2}{|l|}{$\begin{array}{l}\text { Identification accuracy (\%) } \\
\text { using wavelet + DLDA }\end{array}$} \\
\cline { 2 - 5 } & Haar & Db4 & Haar + DLDA & Db4+ DLDA \\
\hline $\begin{array}{c}\text { Iris } \\
\text { Region }\end{array}$ & 89.63 & 93.26 & 97.40 & 97.44 \\
\hline $\begin{array}{c}\text { Collarette } \\
\text { region }\end{array}$ & 89.56 & 94.81 & 98.44 & 98.96 \\
\hline
\end{tabular}

\section{REFERENCES}

[1] J.G. Daugman. The importance of being random: Statistical principles of iris recognition. Pattern recognition, 36(2), (2003) 279-291

[2] J. G. Daugman, "How iris recognition works", IEEE Trans. on circuits and Systems for Video Technology, vol. 14 , no. 1 , January 2004, pp. 21-30

[3] W. Zhao, R. Chellappa, P. J. Phillips, and A. Rosenfeld. Face recognition: A literature survey. ACM Computing Surveys, 35(4):399-458, 2003.

[4] John Daugman. Biometric personal identification system based on iris analysis. U.S. Patent No. 5,291,560, Mar 1994.

[5] T. Chuan Chen, K. Liang Chung: An Efficient Randomized Algorithm for Detecting Circles. Computer Vision and Image Understanding Vol. 83 (2001) 172-191

[6] J. Canny: A Computational Approach to Edge Detection. IEEE Transaction on Pattern Analysis and Machine Intelligence Vol. 8 (1986) 679-714

[7] A Direct LDA Algorithm for High-Dimensional Data with Application to Face Recognition Hua $\mathrm{Yu} \mathrm{1}$, Jie Yang Interactive System Labs, Carnegie Mellon University, Pittsburgh, PA 15213.

[8] D. Swets and J. Weng. Using discriminant eigenfeatures for image retrieval. PAMI, 18(8):831-836, August 1996.

[9] L. Chen, H. Liao, M. Ko, J. Lin, and G. Yu. A new ldabased face recognition system which can solve the small sample size problem. Pattern Recognition, 33(10): $1713\{1726$, Oct 2000

[10] H. Sung, J. Lim, J. Park, and Y. Lee, "Iris recognition using collarette boundary localization". Internat. Conf. on Pattern Recog. vol. 04, 2004.

[11] M. W. Frazier, "An Introduction to Wavelets through Linear algebra", Springer, 1999.

[12] CASIA Iris Image Database. http://www.sinobiometrics.com 
[13] Richard P. Wildes. Iris recognition: an emerging biometric technology. In Proceedings of the IEEE, vol. 85, no.9, pages 1348-1363, U.S.A., September 1997.

[14] Abhishek Gangwar, Akanksha Joshi, Renu Sharma, Zia Saquib, "Robust Iris Templates for Efficient Person Identification" Internat. Conf. on Signal, Image Processing and Pattern Recognition, Springer 2012.

[15] K. Roy and P. Bhattacharya "An Iris Recognition Method based on Zigzag Collarette Area and Asymmetrical Support Vector Machines", in IEEE conference on Systems, Man, and Cybernetics
(SMC'2006), 8-11 October, 2006, Taiwan. Pages 861865.

[16] W. Boles and B. Boashash, "Human Identification Technique Using Images of the Iris and Wavelet Transform", IEEE Trans. on Signal Processing, vol. 46, no. 4, pp. 1185-1188, 1998.

[17] Hanho Sung; Jaekyung Lim; Ji-hyun Park; Yillbyung Lee; , "Iris recognition using collarette boundary localization," Pattern Recognition, 2004. ICPR 2004. Proceedings of the 17th International Conference on, vol.4, no., pp. 857- 860 Vol.4, 23-26 Aug. 2004 doi: 10.1109/ICPR.2004.1333907. 